\title{
CÁC NHÂN TỐ TÁC ĐộNG LợI ÍCH KẾ TOÁN TRONG MÔI TRƯờNG ERP GIAI ĐOẠN SAU TRIỀN KHAI: VAI TRÒ TRUNG GIAN CỦA SỬ HÀI LÒNG NGƯỜI SỬ DỤNG
}

\author{
VÕ VĂN NHI! ${ }^{1}$, ĐÀO NHẬT MINH ${ }^{2}$ \\ ${ }^{1}$ Đại học kinh tế thành phố Hồ Chí Minh, ${ }^{2}$ Đại học Quy Nhơn \\ nhi_vo1958@yahoo.com,daonhatminh@qnu.edu.vn
}

Tóm tắt. Dựa vào mô hình sự thành công hệ thống thông tin của $\mathrm{D}$ \& M (2003), chúng tôi đề xuất chất lượng hệ thống ERP, chất lượng thông tin kế toán trong môi trường ERP và chât lượng dịch vụ của nhà cung cấp giải pháp ERP có tác động trực tiếp và gián tiếp (thông qua sự hài lòng người sử dụng hệ thống ERP) tới các lợi ích kế toán trong môi trường ERP ở giai đoạn sau triển khai hệ thống ERP. Thực hiện kiểm định SEM với mẫu 282 doanh nghiệp đã thực hiện hệ thống ERP từ 1 năm trở lên, kết quả nghiên cứu đã xác nhận sự hài lòng của người sử dụng hệ thống ERP trong doanh nghiệp làm chức năng trung gian trong các mối quan hệ này. Trong đó, chất lượng hệ thống ERP, chất lượng thông tin kế toán trong môi trường ERP có tác động trực tiếp và gián tiếp, còn chất lượng dịch vụ của nhà cung cấp giải pháp ERP chỉ có tác động gián tiếp tới các lợi ích kế toán trong môi trường ERP thông qua biến trung gian sự hài lòng của người sử dụng. Kết quả nghiên cứu giúp các doanh nghiệp nhận ra các nhân tố nào cần chú ý trong giai đoạn sau triển khai để duy trì, nâng cao lợi ích kế toán hệ thống ERP đem lại cho doanh nghiệp mình, trong đó đặc biệt cần chú ý sự hài lòng người sử dụng hệ thống ERP tại doanh nghiệp.

Từ khóa. ERP, sự hài lòng người sử dụng hệ thống ERP, biến trung gian, các lợi ích kế toán trong môi trường ERP, giai đoạn sau triển khai hệ thổng ERP.

\section{THE FACTORS IMPACT ON ACCOUNTING BENEFITS IN ERP ENVIRONMENT IN THE POST-IMPLEMENTATION PHASE: THE MEDIATING ROLE OF}

\section{USER SATISFACTION}

\begin{abstract}
Based on the Model of Information systems success of DeLone and McLean (2003), we have assumed ERP system quality, Accounting information quality in the ERP environment, the quality of ERP vendor's service to affect directly and indirectly accounting benefits through the ERP's user satisfaction in the post-implementation phase of ERP. An empirical test was conducted by SEM with a sample of 282 firms that have implemented an ERP system over 1 year, the results confirmed that the ERP's user satisfaction played a mediating role in these relationships. In particular, ERP system quality and Accounting information quality have impacted directly as well as indirectly on accounting benefits in the ERP environment. On the other hand, The quality of ERP vendor's service has only impacted indirectly on the dependent variable through the ERP's user satisfaction mediating variable. The research results help the firms realize which factors they need to focus on the post-implementation phase to maintain, advance accounting benefits that ERP brings in their firms, therein especially the ERP's user satisfaction.
\end{abstract}

Keywords. ERP, the ERP's user satisfaction, mediating variable, accounting benefits in the ERP environment, the post-implementation phase. 


\section{GIỚI THIẸU}

Mặc dù có nhiều định nghĩa về hệ thống ERP, tuy nhiên không có quá nhiều sự khác biệt giữa các định nghĩa này (M. Al-Mashari và cộng sự, 2003). Theo Rashid \& Cộng sự (2002), hệ thống hoạch định nguồn lực doanh nghiệp hoặc hệ thống doanh nghiệp là hệ thống phần mềm để quản lý doanh nghiệp, bao gồm các module hỗ trợ các lĩnh vực chức năng như lập kế hoạch, sản xuất, bán hàng, tiếp thị, phân phối, kế toán, tài chính, quản lý nhân sự, quản lý dự án, quản lý hàng tồn kho, dịch vụ và bảo trì, vận chuyển và kinh doanh điện tử.

Vòng đời của hệ thống ERP gồm 3 giai đoạn: Giai đoạn trước khi triển khai hệ thống đề cập đến hoạt động quyết định chấp nhận hệ thống và mua sắm; Giai đoạn triển khai hệ thống bao gồm việc tùy biến, điều chỉnh hệ thống để giúp hệ thống ERP mua về phù hợp với yêu cầu của doanh nghiệp và đưa hệ thống vào hoạt động; Và cuối cùng là giai đoạn sau triển khai đề cập đến hoạt động sử dụng và bảo trì hệ thống, hoạt động đổi mới và kết thúc cũng thuộc giai đoạn này (Jose M. Esteves và Joan A. Pastor, 1999). Trong nghiên cứu này, khi đề cập đến giai đoạn sau triển khai, nhóm tác giả muốn nói tới giai đoạn sử dụng và bảo trì hệ thống.

Tại Việt Nam, nhiều công ty, tập đoàn lớn đã thực hiện hệ thống ERP từ lâu như: MWG, Vinamilk hay Công ty Cổ phần Cảng Đồng Nai.... Thời gian gần đây, các doanh nghiệp có quy mô vừa cũng bắt đầu thực hiện các giải pháp ERP vì hệ thống ERP được đánh giá là sẽ tăng khả năng quản lý, ra quyết định cho doanh nghiệp dù ở quy mô nào, giúp doanh nghiệp tồn tại và phát triển. Các giải pháp ERP được các công ty này lựa chọn thường là các giải pháp ERP đến từ các nhà cung cấp Việt Nam, hệ thống ERP được triển khai tại các doanh nghiệp có quy mô vừa thường là phần mềm ERP đóng gói, vì những ưu điểm về chi phí cũng như công tác triển khai.

Tuy nhiên, hầu hết các công ty Việt Nam đều chỉ tập trung vào giai đoạn triển khai hệ thống ERP, mà thường không quan tâm đến các vấn đề sau triển khai. Trong khi đó, giai đoạn sau triển khai lại là giai đoạn có thể nâng cao hoặc làm giảm những lợi ích tổng thể hay lợi ích kế toán mà hệ thống ERP đem lại cho doanh nghiệp bất kể giai đoạn triển khai đã thành công như thế nào. Tại nhiều công ty, sau khi đã triển khai hệ thống ERP được vài năm, việc thành công ở giai đoạn triển khai ban đầu đã không giúp duy trì được các lợi ích mà doanh nghiệp cần ở hệ thống ERP giai đoạn sau triển khai (Shih-Wei Chou và Yu-Chieh Chang, 2008).

Nghiên cứu về các lợi ích hệ thống ERP đem lại cho doanh nghiệp là quan trọng, trong đó lợi ích kế toán là vấn đề không thể bỏ qua, vì hệ thống thông tin kế toán được xem là trái tim của hệ thống ERP. Tuy nhiên, dựa vào việc tổng quan tài liệu, các nghiên cứu trên thế giới thường trình bày chung lợi ích kế toán trong các lợi ích tổng thể mà doanh nghiệp nhận được từ hệ thống ERP, số lượng tác giả tập trung vào lợi ích kế toán rất ít.

Spathis và Constantinides (2004) đã chỉ ra sự thay đổi lợi ích quan trọng nhất đối với hoạt động kế toán khi triển khai hệ thống ERP đó là hệ thống ERP giúp thổng tin kế toán trong doanh nghiệp linh hoạt hơn, nâng cao khả năng tích hợp của các ứng dụng kế toán, nâng cao chất lượng và thời gian của các báo cáo kế toán, thông tin kế toán đáng tin cậy và kịp thời hơn.

Spathis và Ananiadis (2005) thực hiện đánh giá tại một trường đại học công lập Hy Lạp về thông tin kế toán và lợi ích trong việc quản lý khi áp dụng hệ thổng ERP đã xác nhận việc cung cấp thông tin sẽ linh hoạt hơn rất nhiều sau khi thực hiện một hệ thống ERP, điều này có được nhờ việc khai thác và giám sát có hiệu quả các tài sản của trường đại học và quá trình luân chuyển doanh thu - chi phí, cải thiện khả năng ra quyết định.

Colmenares (2009) thực hiện một nghiên cứu xem xét trong một doanh nghiệp lớn tại Venetuela về mức độ ảnh hưởng của việc sử dụng hệ hống ERP đến các mức độ quản trị khác nhau của quản trị tài chính và kế toán. Kết quả cho thấy ERP mang lại một số lợi ích đối với việc quản trị tài chính và kế toán đó là: cải thiện việc kiểm soát và giám sát nhân viên, nâng cao tính chính xác của báo cáo tài chính, cải thiện quá trình ra quyết định quản trị, tăng cường khả năng hội nhập của doanh nghiệp .

Despina Galani, Efthymios Gravas, Antonios Stavropoulos (2010) chỉ ra việc thực hiện ERP sẽ đem lại lợi ích cao hơn về chất lượng thông tin doanh nghiệp như chất lượng thông tin kế toán.

Alexandra Kanellou, Charalambos Spathis (2013) đã chỉ ra những lợi ích kế toán mà doanh nghiệp nhận được khi triển khai hệ thống ERP, phân loại những lợi ích kế toán này, tìm hiểu sự tác động của lợi ích kế toán đến sự hài lòng của người sử dụng hệ thống ERP, chứ chưa tìm hiểu chiều ngược lại. Đồng thời, 
đơn vị phân tích của nghiên cứu này là đơn vị cá nhân, chưa tìm hiểu đơn vị tổ chức. Một bài báo khác được trình bày trên Tạp chí quản lý thông tin doanh nghiệp vào năm 2006, với tựa đề "Việc triển khai hệ thống ERP và các lợi ích kế toán" của Charalambos Spathis đã chỉ ra các nhân tố của việc triển khai hệ thống ERP tác động đến các lợi ích kế toán đó là: nguyên nhân việc triển khai hệ thống ERP, số lượng các module hệ thống, chi phí của việc triển khai hệ thống theo phần trăm doanh thu và tổng tài sản của công ty. Nhưng ở giai đoạn sau triển khai hệ thống, khi hệ thống ERP đã đi vào hoạt động, thì các nhân tố nào sẽ ảnh hưởng đến các lợi ích kế toán? Đây là vấn đề rất đáng quan tâm. Một số nghiên cứu đề xuất rằng trong giai đoạn sau triển khai, chất lượng hệ thống ERP và chất lượng thông tin do hệ thống ERP cung cấp (Paul Hawking và cộng sự, 2004; Edith Galy và Mary Jane Sauceda, 2014); Pedro Ruivo và cộng sự, 2014) cũng như chất lượng dịch vụ của nhà cung cấp giải pháp ERP (Jiwat Ram và cộng sự, 2013; Young Mok Haa và Hyung Jun Ahn, 2014) có tác động đến lợi ích doanh nghiệp trong hệ thống ERP; hay hiệu quả ERP; giá trị ERP đem đến cho doanh nghiệp. Nên nhiều khả năng các nhân tố này sẽ tác động đến các lợi ích kế toán hệ thống ERP đem đến cho doanh nghiệp giai đoạn sau triển khai.

Một vấn đề khác các nhà nghiên cứu quan tâm đó là chất lượng hệ thống ERP, chất lượng thông tin do hệ thống ERP cung cấp, và chất lượng dịch vụ của nhà cung cấp giải pháp ERP sẽ có tác động trực tiếp đến lợi ích kế toán hay phải qua một số trung gian khác?

Nghiên cứu này dựa vào mô hình $\mathrm{D} \& \mathrm{M}$ (2003), giả thuyết rằng chất lượng hệ thống ERP, chất lượng thông tin kế toán do hệ thống ERP cung cấp (vì hướng nghiên cứu của luận án là lợi ích kế toán), và chất lượng dịch vụ của nhà cung cấp giải pháp ERP có tác động trực tiếp và gián tiếp (thông qua sự hài lòng người sử dụng hệ thống $\mathrm{ERP}$ ) vào các lợi ích kế toán trong môi trường ERP giai đoạn sau triển khai. Các phần tiếp theo của nghiên cứu sẽ trình bày cơ sở lý thuyết và giả thuyết nghiên cứu, phương pháp nghiên cứu, kết quả và hàm ý của nghiên cứu.

\section{CƠ SỞ LÝ THUYẾT VÀ GIẢ THUYẾT NGHIÊN CÚ̉U}

\subsection{Cơ sở lý thuyết}

Dựa vào mô hình sự thành công của hệ thống thông tin (Model of Information systems success) của DeLone and McLean (2003), nhóm tác giả đề xuất mô hình nghiên cứu (hình 1). Bắt nguồn từ lý thuyết giao tiếp của Shannon và Weaver (1949), sự phân loại hệ thống thông tin của của Mason (1978) cùng với việc tổng quan các tài liệu liên quan, DeLone và McLean (1992) đã đề xuất một mô hình các nhân tố quan trọng đo lường sự thành công của một hệ thống thông tin. Sau đó, cùng với việc thay đổi của môi trường thông tin xã hội, và sự góp ý từ các nhà nghiên cứu khác, mô hình này đã được cập nhập và trở nên rất phổ biến, được đặt tên là mô hình sự thành công của hệ thống thông tin cập nhập D \& M (2003).

So với mô hình D \& M (2003), mô hình nghiên cứu nhóm tác giả đề xuất có một số sự thay đổi. Đó là mặc dù trên mô hình $\mathrm{D} \& \mathrm{M}$ (2003) không thể hiện sự tác động của chất lượng hệ thống, chất lượng thông tin và chất lượng dịch vụ tới lợi ích thuần, nhưng Delone và McLean (2016) đã đề nghị việc ứng dụng mô hình $\mathrm{D} \& \mathrm{M}$ trong thực tế cần dựa vào bối cảnh tổ chức và việc lựa chọn các chiều tác động cụ thể sẽ dựa vào tính chất và mục đích của hệ thống được đánh giá. Đồng thời, Stacie Petter và cộng sự (2008) đã cho rằng lý tưởng nhất là mô hình $\mathrm{D} \& \mathrm{M}$ được xem xét ở nhiều bối cảnh (cá nhân và tổ chức) và nhiều chiều tác động.

Nhân tố sử dụng/ ý định sử dụng sẽ được bỏ ra khỏi mô hình vì theo Stacie Petter và cộng sự (2008), ý định sử dụng thường được sử dụng ở đơn vị phân tích cá nhân, không thích hợp cho các nghiên cứu sử dụng đơn vị phân tích tổ chức như nghiên cứu. Đồng thời, theo nhiều nhà nghiên cứu, nhân tố sử dụng nên được bỏ ra khỏi mô hình $\mathrm{D} \& \mathrm{M}$ nhất là khi việc sử dụng hệ thống là bắt buộc (DeLone \& McLean, 2016).

Còn sự hài lòng của người sử dụng, nếu được đo lường bởi các nhà quản lý chức năng mà ở đây là quản lý hệ thống thông tin kể toán trong môi trường ERP sẽ đại diện cho sự hài lòng ở cấp tổ chức (Marianne Bradford và cộng sự, 2003), do đó với đơn vị phân tích tổ chức như nghiên cứu thực hiện, nhân tố này vẫn có thể có sự tác động đến lợi ích kế toán trong môi trường ERP.

Khái niệm lợi ích kế toán trong môi trường ERP thì chưa được thống nhất trong các nghiên cứu về ERP, nhưng có thể được khái quát như sau: Lợi ích kế toán trong môi trương ERP là nhũng điều có ích, nhũng tác động tích cực mà hệ thống ERP đem lại cho hệ thống thông tin kế toán của doanh nghiẹp. Như vậy, trong nghiên cứu này, khi nói về lợi ích kế toán trong môi trường ERP, tác giả muốn nói tới những lợi ích về mặt kế toán mà doanh nghiệp nhận được từ hệ thống ERP. 


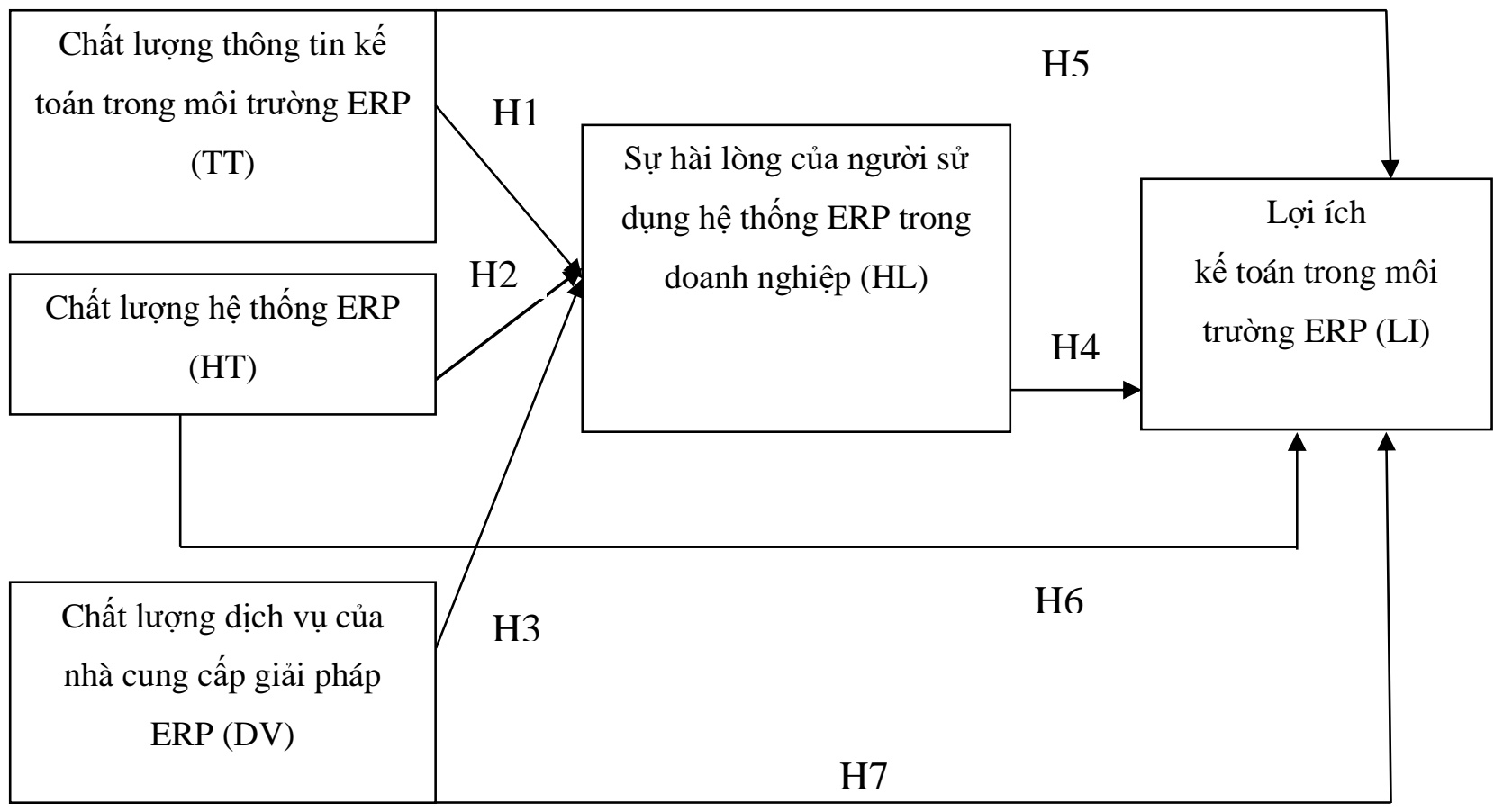

Hình 1. Mô hình nghiên cứu đề xuất

\subsection{Giả thuyết nghiên cứu}

Trên cơ sở mô hình nghiên cứu đề xuất, nhóm tác giả đưa ra một số giả thuyết nghiên cứu như sau: H1: Chất lượng thông tin kế toán có sự tác động dương đến Sự hài lòng của người sử dụng hệ thống ERP. H2: Chất lượng hệ thống ERP có sự tác động dương đến Sự hài lòng của người sử dụng hệ thống ERP.

H3: Chất lượng dịch vụ của nhà cung cấp giải pháp ERP có sự tác động dương đến Sự hài lòng của người sử dụng hệ thống ERP.

H4: Sự hài lòng của người sử dụng hệ thống ERP có sự tác động dương đến các lợi ích kế toán trong môi trường ERP.

H5: Chất lượng thông tin kế toán có sự tác động dương đến các lợi ích kế toán trong môi trường ERP.

H6: Chất lượng hệ thống ERP có sự tác động dương đến các lợi ích kế toán trong môi trường ERP.

H7: Chất lượng dịch vụ của nhà cung cấp giải pháp ERP có sự tác động dương đến các lợi ích kế toán trong môi trường ERP.

\section{PHƯƠNG PHÁP NGHIÊN CÚU}

\subsection{Mẫu}

Vì khó xác định chính xác các doanh nghiệp đang áp dụng ERP nên nhóm tác giả chọn phương pháp lấy mẫu thuận tiện kết hợp với phương pháp phát triển mầm. Thông thường nghiên cứu định lượng đòi hỏi mẫu có kích thước lớn và thường được chọn theo phương pháp xác xuất để có thể đại diện được cho đám đông cần nghiên cứu. Tuy nhiên, việc chọn mẫu phi xác suất cũng được sử dụng khá phổ biến trong nghiên cứu định lượng bởi nếu trong quá trình kiểm định mà dữ liệu của mẫu này không bị từ chối thì kiểm định này vẫn đóng góp phần đánh giá lý thuyết đó (Nguyễn Đình Thọ, 2014).Tổng cộng 300 phiếu khảo sát đã 
được gởi đi với thời gian từ tháng 6/2018 - 12/2018, sau khi thu thập và kiểm tra thì 18 phiếu bị loại do cung cấp thông tin không đồng nhất, doanh nghiệp không phù hợp để tham gia khảo sát hoặc trả lời không đủ số câu hỏi. Trong tổng số 282 doanh nghiệp được khảo sát phù hợp, 100 doanh nghiệp tại Bình Định (35,46\%), 150 doanh nghiệp tại thành phố Hồ Chí Minh $(53,19 \%)$ và 32 doanh nghiệp tại Đà Nẵng $(11,35 \%)$. Lĩnh vực hoạt động của các doanh nghiệp này bao gồm: Sản xuất $(51 \%)$, thương mại $(29 \%)$ và dịch vụ $(20 \%)$. Đa phần quy mô của các doanh nghiệp này là vừa $(74 \%)$, còn lại là các doanh nghiệp có quy mô lớn (26\%). Về loại hình doanh nghiệp, các công ty cổ phần chiếm $68 \%$ các doanh nghiệp được khảo sát, các công ty trách nhiệm hữu hạn chiếm $32 \%$.

Với người trực tiếp trả lời bảng khảo sát tại các doanh nghiệp, thì đa số là các kế toán trưởng (78\%), còn lại là giám đốc $(22 \%)$. Số năm kinh nghiệm trong sử dụng hệ thống ERP của các đối tượng này đều từ 3 năm trở lên. Cơ cấu giới tính chủ yếu là nam $(65 \%)$, nữ (35\%). Độ tuổi lớn hơn 30 chiếm tỷ lệ lớn $80 \%$, dưới 30 tuổi là $20 \%$.

3.2 Thang đo

Có 5 khái niệm nghiên cứu, đó là Chất lượng thông tin kế toán trong môi trường ERP; Chất lượng hệ thống ERP; Chất lượng dịch vụ của nhà cung cấp giải pháp ERP; Sự hài lòng của người sử dụng hệ thống ERP trong doanh nghiệp; và lợi ích kế toán trong môi trường ERP. Thang đo Chất lượng thông tin kế toán trong môi trường ERP gồm 9 biến quan sát, dựa theo thang đo của DeLone và McLean (2016) cùng với Sedera và cộng sự (2004). Thang đo Chất lượng hệ thống ERP cũng dựa theo thang đo của DeLone và McLean (2016) cùng với Sedera và cộng sự (2004), gồm 12 biến quan sát. Thang đo Chất lượng dịch vụ của nhà cung cấp giải pháp ERP dựa theo thang đo của DeLone và McLean (2016) gồm 5 biến quan sát. Thang đo Sự hài lòng của người sử dụng hệ thống ERP trong doanh nghiệp dựa theo thang đo của Bradford và Florin (2003) gồm 1 biến quan sát. Và cuối cùng, thang đo lợi ích kế toán trong môi trường ERP dựa vào thang đo của Alexandra Kanellou, Charalambos Spathis (2013) là thang đo bậc 2 gồm 5 thang đo bậc 1: lợi ích kế toán về mặt CNTT (LI-CN 5 biến quan sát), lợi ích kế toán về mặt hoạt động (thời gian) (LITG 2 biến quan sát), lợi ích kế toán về mặt vận hành (LI_TC 5 biến quan sát), lợi ích kế toán quản trị (LIKTQT 3 biến quan sát), và lợi ích kế toán về mặt hoạt động (chi phí) (LI-CP 1 biến quan sát). Các biến quan sát được đo lường bằng thang đo Likert 5 điểm, với 1 : hoàn toàn phản đối và 5 : hoàn toàn đồng ý.

Bảng 1. Tổng hợp khái niệm nghiên cứu và thang đo

\begin{tabular}{|c|c|c|c|}
\hline \multirow{2}{*}{\multicolumn{2}{|c|}{$\begin{array}{l}\text { Khái niệm } \\
\text { nghiên cứu }\end{array}$}} & \multicolumn{2}{|l|}{ Thang đo } \\
\hline & & Nội dung & Mã hóa \\
\hline \multirow{8}{*}{$\begin{array}{l}\text { LIKT } \\
\text { trong hệ } \\
\text { thống } \\
\text { ERP }\end{array}$} & \multirow{5}{*}{$\begin{array}{c}\text { (LI-CN) } \\
\text { LIKT về mặt } \\
\text { CNTT }\end{array}$} & ERP thu thập dữ liệu nhanh hơn & LI-CN1 \\
\hline & & ERP tổng hợp dữ liệu dễ dàng hơn & LI-CN2 \\
\hline & & ERP tạo ra thông tin nhanh hơn & LI-CN3 \\
\hline & & ERP tạo ra thông tin dễ dàng hơn & LI-CN4 \\
\hline & & $\begin{array}{c}\text { Nhìn chung, ERP là linh hoạt hơn trong việc thu thập dữ liệu } \\
\text { và tạo ra thông tin }\end{array}$ & LI-CN5 \\
\hline & \multirow{2}{*}{$\begin{array}{l}\text { (LI-TG) } \\
\text { LIKT về mặt } \\
\text { hoạt động } \\
\text { (thời gian) }\end{array}$} & ERP làm giảm thời gian khóa sổ kế toán & LI-TG1 \\
\hline & & ERP làm giảm thời gian tạo ra Báo cáo tài chính. & LI-TG2 \\
\hline & & ERP làm tăng tính linh hoạt trong việc truy cập thông tin. & LI-TC1 \\
\hline
\end{tabular}


GIAI ĐOẠN SAU TRIỂN KHAI: VAI TRÒ̉ TRUNG GIAN CỦA SỬ HÀI LÒNG NGƯỜI SỦ DỬ

\begin{tabular}{|c|c|c|}
\hline \multirow{4}{*}{$\begin{array}{c}\text { (LI-TC) } \\
\text { LIKT về mặt } \\
\text { vận hành }\end{array}$} & $\begin{array}{c}\text { ERP làm tăng sự tích hợp của các ứng dụng liên quan kế } \\
\text { toán. }\end{array}$ & LI-TC2 \\
\hline & $\begin{array}{l}\text { ERP cải thiện việc ra quyết định dựa trên thông tin kịp thời } \\
\text { và đáng tin cậy. }\end{array}$ & LI-TC3 \\
\hline & ERP cải thiện chất lượng các báo cáo, sổ sách kế toán. & LI-TC4 \\
\hline & ERP làm tăng cường chức năng kiểm toán nội bộ. & LI-TC5 \\
\hline \multirow{3}{*}{$\begin{array}{l}\text { (LI-KTQT) } \\
\text { LIKT quản } \\
\quad \text { trị }\end{array}$} & ERP làm tăng cường khả năng kiểm soát tài sản ngắn hạn. & $\begin{array}{c}\text { LI- } \\
\text { KTQT1 }\end{array}$ \\
\hline & $\begin{array}{c}\text { ERP làm tăng cường sử dụng việc phân tích các chỉ số tài } \\
\text { chính. }\end{array}$ & $\begin{array}{l}\text { LI- } \\
\text { KTQT2 }\end{array}$ \\
\hline & ERP làm giảm thời gian phát hành bảng lương. & $\begin{array}{l}\text { LI- } \\
\text { KTQT3 }\end{array}$ \\
\hline $\begin{array}{l}\text { (LI-CP) } \\
\text { LIKT về mặt } \\
\text { hoạt động } \\
\text { (chi phí) }\end{array}$ & ERP làm giảm nhân sự phòng kế toán & LI-CP1 \\
\hline \multirow{9}{*}{ CLTT kế toán } & $\begin{array}{l}\text { Hệ thống ERP cung cấp các báo cáo đầu ra liên quan tới } \\
\text { những gì công ty tôi cần. }\end{array}$ & TT1 \\
\hline & $\begin{array}{c}\text { Thông tin kế toán do hệ thống ERP cung cấp rất hữu ích đối } \\
\text { với công ty tôi. }\end{array}$ & TT2 \\
\hline & Thông tin kế toán do hệ thống ERP cung cấp rất dễ hiểu. & TT3 \\
\hline & $\begin{array}{c}\text { Thông tin kế toán do hệ thống ERP cung cấp có tính chính } \\
\text { xác cao. }\end{array}$ & TT4 \\
\hline & $\begin{array}{c}\text { Thông tin kế toán do hệ thống ERP cung cấp có độ tin cậy } \\
\text { cao. }\end{array}$ & TT5 \\
\hline & $\begin{array}{c}\text { Thông tin kế toán do hệ thống ERP cung cấp là thông dụng } \\
\text { với công ty tôi. }\end{array}$ & TT6 \\
\hline & $\begin{array}{l}\text { Thông tin kế toán do hệ thống ERP cung cấp luôn ở định } \\
\text { dạng hoàn thiện. }\end{array}$ & TT7 \\
\hline & $\begin{array}{c}\text { Thông tin kế toán do hệ thống ERP cung cấp luôn có sẵn, kịp } \\
\text { thời, đúng lúc công ty tôi cần. }\end{array}$ & TT8 \\
\hline & $\begin{array}{c}\text { Thông tin kế toán do hệ thống ERP cung cấp luôn đầy đủ, súc } \\
\text { tích }\end{array}$ & TT9 \\
\hline
\end{tabular}




\begin{tabular}{|c|c|c|}
\hline & Hệ thống ERP dễ sử dụng. & HT1 \\
\hline & Hệ thống ERP dễ học hỏi. & HT2 \\
\hline & Hệ thống ERP có độ tin cậy cao. & HT3 \\
\hline & $\begin{array}{l}\text { Hệ thống ERP có thể dễ dàng tùy chỉnh hoặc cải tiến theo } \\
\text { yêu cầu của công ty tôi. }\end{array}$ & HT4 \\
\hline & Hệ thống ERP có thể linh hoạt thay đổi theo các yêu cầu mới. & HT5 \\
\hline & $\begin{array}{l}\text { Thời gian cho các thao tác, xử lý dữ liệu của hệ thống ERP } \\
\text { nhanh. }\end{array}$ & HT6 \\
\hline CLHT ERP & Hệ thống ERP đáp ứng công việc của công ty tôi. & HT7 \\
\hline & $\begin{array}{c}\text { Giao diện làm việc của hệ thống ERP thuận lợi cho việc } \\
\text { tương tác với hệ thống. }\end{array}$ & HT8 \\
\hline & Hệ thống ERP có tính bảo mật cao. & HT9 \\
\hline & $\begin{array}{l}\text { Hệ thống ERP có độ chính xác cao trong các thao tác, xử lý } \\
\text { dữ liệu. }\end{array}$ & HT10 \\
\hline & $\begin{array}{c}\text { Dữ liệu của hệ thống ERP có sự tích hợp tốt giữa các bộ phận } \\
\text { trong doanh nghiệp. }\end{array}$ & HT11 \\
\hline & $\begin{array}{l}\text { Hệ thống ERP có đầy đủ tính năng cần thiết cho công việc } \\
\text { của công ty tôi }\end{array}$ & HT12 \\
\hline & $\begin{array}{l}\text { Nhà cung cấp giải pháp ERP thực hiện đúng các yêu cầu như } \\
\text { họ đã cam kết. }\end{array}$ & DV1 \\
\hline & $\begin{array}{l}\text { Nhà cung cấp giải pháp ERP hiểu rõ những nhu cầu của công } \\
\text { ty tôi. }\end{array}$ & DV2 \\
\hline $\begin{array}{l}\text { CLDV của nhà cung cấp } \\
\text { giải pháp ERP }\end{array}$ & $\begin{array}{c}\text { Nhà cung cấp giải pháp ERP luôn trợ giúp, hồi đáp nhanh } \\
\text { chóng các vướng mắc của công ty tôi trong quá trình sử } \\
\text { dụng. }\end{array}$ & DV3 \\
\hline & $\begin{array}{l}\text { Nhà cung cấp giải pháp ERP có rất nhiều kênh như: đường } \\
\text { dây nóng, mail, facebook...để liên lạc. }\end{array}$ & DV4 \\
\hline & $\begin{array}{l}\text { Việc phối hợp, tương tác giữa nhà cung cấp giải pháp ERP và } \\
\text { công ty tôi trong thực hiện công việc rất hiệu quả. }\end{array}$ & DV5 \\
\hline $\begin{array}{l}\text { Sự hài lòng của người sử } \\
\text { dụng trong hệ thống ERP }\end{array}$ & $\begin{array}{l}\text { Quản lý bộ phận kế toán tại công ty hài lòng với hệ thống ERP } \\
\text { được công ty thực hiện. }\end{array}$ & HL1 \\
\hline
\end{tabular}




\section{KẾT QUẢ KIỂM ĐỊNH GIẢ THUYẾT}

\section{1 Đánh giá thang đo}

Độ tin cậy Cronbach alpha và phương pháp phân tích nhân tố khám phá EFA (exploratory factor analysis) được sử dụng để đánh giá các thang đo. Kết quả Cronbach alpha cho thấy độ tin cậy các thang đo đều chấp nhận được: $\mathrm{a}_{\mathrm{TT}}=0,905 ; \mathrm{a}_{\mathrm{HT}}=0,958 ; \mathrm{a}_{\mathrm{DV}}=0,890 ; \mathrm{a}_{\mathrm{HL}}=0,785$. Các thang đo bậc 1 của thang đo bậc 2 lợi ích kế toán trong môi trường ERP độ tin cậy cũng đạt yêu cầu: $\mathrm{a}_{\mathrm{LI}-\mathrm{CN}}=0,886 ; \mathrm{a}_{\mathrm{LI}-\mathrm{TC}}=0,811$; $\mathrm{a}_{\mathrm{LI}}$ ктQт $=0,882$. Kết quả EFA cho thấy cho thấy các thang đo đạt yêu cầu nhân tố trích, tổng phương sai trích và trọng số nhân tố của các biến quan sát: thang đo bậc hai lợi ích kế toán trong môi trường ERP có $0,5<$ $\mathrm{KMO}=0,568<1, \mathrm{Sig}=0,000<0,05$, eigenvalue 1,038 với phương sai trích $(\%$ cumulative variance) là $76,101 \%$. Thực hiện $\mathrm{EFA}$ với các khái niệm nghiên cứu còn lại, kết quả cho thấy $0,5<\mathrm{KMO}=0,789<1$, Sig $=0,000<0,05$, eigenvalue 1,598 với phương sai trích (\% cumulative variance) là $73,938 \%$. Và trọng số $E F A$ đều $\geq 0.55$.

\subsection{Kết quả kiểm định giả thuyết}

Bảng 2. Tác động trục tiếp và gián tiếp của các nhân tố

\begin{tabular}{|c|c|c|c|c|c|c|c|}
\hline \multicolumn{3}{|c|}{ Quan hệ } & $\mathbf{P}$ & Tác động & & Giải thích tác động & Tổng công \\
\hline HL & $<--$ & HT & $* * *$ & 0,216 & 0,000 & & 0,216 \\
\hline HL & $<--$ & $\mathrm{TT}$ & 0,003 & 0,155 & 0,000 & & 0,155 \\
\hline HL & $<--$ & DV & $* * *$ & 0,383 & 0,000 & & 0,383 \\
\hline LI & $<--$ & $\mathrm{HL}$ & 0,031 & 0,152 & 0,000 & & 0,152 \\
\hline LI & $<--$ & HT & 0,013 & 0,133 & 0,033 & $\begin{array}{c}\mathrm{HT} \rightarrow \mathrm{HL} \rightarrow \mathrm{LI}(0,216 \\
\mathrm{x} \mathrm{0,152)}\end{array}$ & 0,166 \\
\hline LI & $<--$ & TT & 0,006 & 0,129 & 0,023 & $\begin{array}{l}\mathrm{TT} \rightarrow \mathrm{HL} \rightarrow \mathrm{LI} \\
(0,155 \times 0,152)\end{array}$ & 0,152 \\
\hline LI & $<--$ & DV & 0,055 & 0,114 & 0,06 & $\begin{array}{l}\mathrm{DV} \rightarrow \mathrm{HL} \rightarrow \mathrm{LI} \\
(0,383 \times 0,152)\end{array}$ & 0,174 \\
\hline
\end{tabular}

Ghi chú: $* * *=0,000$

(Nguồn: nhóm tác giả tụ tổng hợp)

Trong bảng 2, sử dụng kỹ thuật phân tích đường dẫn (PATH) trong mô hình SEM, tác động trực tiếp là hệ số hồi quy giữa biến độc lập và biến phụ thuộc trong mô hình nghiên cứu, tác động gián tiếp là tác động giữa biến độc lập và biển phụ thuộc thông qua biến trung gian và được tính bằng cách nhân các hệ số của các biến trên cùng đường dẫn. Cột tổng cộng là tổng hợp mối quan hệ trực tiếp và gián tiếp của các biến độc lập tới biến phụ thuộc (Nguyễn Đình Thọ, 2014).

Ở giai đoạn sau triển khai, với sự tác động của Chất lượng thông tin kế toán đến Lợi ích kế toán trong môi trường ERP, tất cả các mối quan hệ bao gồm: Chất lượng thông tin kế toán đến Sự hài lòng của người sử dụng trong hệ thống ERP, Sự hài lòng của người sử dụng trong hệ thống ERP đến Lợi ích kế toán trong môi trường ERP và Chất lượng thông tin kế toán đến Lợi ích kế toán trong môi trường ERP đều có ý nghĩa 
thống kê ( $\mathrm{P}$ - value <=0,05). Mức độ tác động gián tiếp nhỏ hơn mức độ tác động trực tiếp. Như vậy ở giai đoạn sau triển khai, chất lượng thông tin kế toán ngoài việc tác động trực tiếp đến Lợi ích kế toán trong môi trường ERP còn tác động gián tiếp thông qua biến trung gian Sự hài lòng của người sử dụng trong hệ thống ERP, thông qua biến này mức độ ảnh hưởng của Chất lượng thông tin kế toán đến Lợi ích kế toán trong môi trường ERP tăng từ 0,129 lên 0,152 . Như vậy có thể thấy ở giai đoạn sau triển khai, Chất lượng thông tin kế toán thúc đẩy sự hài lòng của người sử dụng trong hệ thống ERP qua đó nâng cao lợi ích kế toán trong môi trường ERP.

Tương tự, ở giai đoạn sau triển khai, với sự tác động của Chất lượng hệ thống ERP đến Lợi ích kế toán trong môi trường ERP, tất cả các mối quan hệ bao gồm: Chất lượng hệ thống ERP đến Sự hài lòng của người sử dụng trong hệ thống ERP, Sự hài lòng của người sử dụng trong hệ thống ERP đến Lợi ích kế toán trong môi trường ERP và Chất lượng hệ thống ERP đến Lợi ích kế toán trong môi trường ERP đều có ý nghĩa thống kê $(\mathrm{P}-$ value $<=0,05)$. Mức độ tác động gián tiếp nhỏ hơn mức độ tác động trực tiếp. Như vậy ở giai đoạn sau triển khai, chất lượng hệ thống ERP ngoài việc tác động trực tiếp đến Lợi ích kế toán trong môi trường ERP còn tác động gián tiếp thông qua biến trung gian Sự hài lòng của người sử dụng trong hệ thống ERP, thông qua biến này mức độ ảnh hưởng của Chất lượng hệ thống ERP đến Lợi ích kế toán trong môi trường ERP tăng từ 0,133 lên 0,166 . Như vậy, có thể thấy trong giai đoạn sau triển khai, Chất lượng hệ thống ERP thúc đẩy sự hài lòng của người sử dụng trong hệ thống ERP qua đó nâng cao lợi ích kế toán trong môi trường ERP.

Đối với sự tác động của nhân tố giai đoạn sau triển khai Chất lượng dịch vụ của nhà cung cấp giải pháp ERP đến Lợi ích kể toán trong môi trường ERP, mối quan hệ trực tiếp giữa biến độc lập và biến phụ thuộc này không có ý nghĩa thống kê $(\mathrm{P}$ - value $>0,05)$. Tuy nhiên, để biến trung gian hiện diện không cần thiết biến độc lập phải có tác động vào biến phụ thuộc. Và lúc này, ta không cần so sánh hệ số hồi quy khi có và không có biến trung gian (Nguyễn Đình Thọ, 2016). Mối quan hệ từ Chất lượng dịch vụ của nhà cung cấp giải pháp ERP đến Sự hài lòng của người sử dụng trong hệ thống ERP và Sự hài lòng của người sử dụng trong hệ thống ERP đến Lợi ích kế toán trong môi trường ERP đều có ý nghĩa thống kê. Do đó, ta có thể kết luận trong giai đoạn sau triển khai, Chất lượng dịch vụ của nhà cung cấp giải pháp ERP không có tác động trực tiếp vào Lợi ích kế toán trong môi trường ERP mà sẽ có tác động gián tiếp vào biến phụ thuộc này thông qua biến trung gian Sự hài lòng của người sử dụng trong hệ thống ERP.

\section{HÀM Ý VÀ KẾT LUẬN}

Mục đích chính của nghiên cứu này là khám phá vai trò trung gian của nhân tố "Sự hài lòng của người sử dụng trong hệ thống ERP” trong sự tác động của các nhân tố giai đoạn sau triển khai hệ thống ERP gồm: (1) "Chất lượng thông tin kế toán", (2) "Chất lượng hệ thống ERP" và (3) "Chất lượng dịch vụ của nhà cung cấp giải pháp ERP" đến "Lợi ích kế toán trong môi trường ERP”. Nhóm tác giả sử dụng phương pháp nghiên cứu định tính kết hợp định lượng, trong đó phương pháp định tính được sử dụng để xây dựng mô hình, giả thuyết nghiên cứu và thang đo các khái niệm nghiên cứu; phương pháp định lượng được sử dụng để kiểm tra các giả thuyết nghiên cứu. Kết quả nghiên cứu cho thấy Chất lượng thông tin kế toán và Chất lượng hệ thống ERP có tác động trực tiếp và gián tiếp (thông qua biến trung gian Sự hài lòng của người sử dụng trong hệ thống ERP) đến Lợi ích kế toán trong môi trường ERP giai đoạn sau triển khai. Trong khi đó, ở giai đoạn sau triển khai Chất lượng dịch vụ của nhà cung cấp giải pháp ERP chỉ có tác động gián tiếp vào biến phụ thuộc Lợi ích kế toán trong môi trường ERP thông qua biến trung gian Sự hài lòng của người sử dụng trong hệ thống ERP. Từ kết quả này, cho chúng ta một số hàm ý về mặt lý thuyết và thực tiễn.

Về mặt lý thuyết, kết quả nghiên cứu đã xác nhận giai đoạn sau triển khai hệ thống ERP là giai đoạn cần tập trung nghiên cứu để đảm bảo duy trì những lợi ích doanh nghiệp nhận được từ hệ thống ERP. Đồng thời, cung cấp bằng chứng thực nghiệm về vai trò trung gian của nhân tố Sự hài lòng của người sử dụng trong hệ thống ERP trong việc thúc đẩy sự tác động của các nhân tố ở giai đoạn sau triển khai: Chất lượng thông tin kế toán; Chất lượng hệ thống ERP; và Chất lượng dịch vụ của nhà cung cấp giải pháp ERP đến các lợi ích kế toán trong môi trường ERP.

Về mặt thực tiễn, kết quả nghiên cứu cho thấy các doanh nghiệp cần chú ý đến sự hài lòng của người sử dụng hệ thống ERP trong doanh nghiệp ở giai đoạn sau triển khai hệ thống ERP. Ngoài việc ảnh hưởng trực tiếp đến các lợi ích kế toán doanh nghiệp có được từ hệ thống ERP, sự hài lòng người sử dụng trong 
GIAI ĐOẠN SAU TRIỂN KHAI: VAI TRÒ TRUNG GIAN CỦA SỰ HÀI LÒNG NGƯỜI SỦ DỤNG

hệ thống ERP còn đóng vai trò trung gian, tăng cường mức độ tác động của các nhân tố chất lượng hệ thống ERP, chất lượng thông tin kế toán đến các lợi ích kế toán hệ thống ERP đem lại cho doanh nghiệp. Do đó, có thể thấy ở giai đoạn sau triển khai, vai trò sự hài lòng của người sử dụng hệ thống ERP trong việc duy trì, nâng cao lợi ích kế toán doanh nghiệp nhận được từ hệ thống ERP là vô cùng to lớn. Đồng thời, trong giai đoạn sau triển khai, chất lượng dịch vụ của nhà cung cấp giải pháp ERP chỉ có tác động gián tiếp đến các lợi ích kế toán trong hệ thống ERP thông qua sự hài lòng người sử dụng trong hệ thống ERP, chứ không có tác động trực tiếp. Vì vậy, trong giai đoạn sau triển khai hệ thống ERP, các doanh nghiệp cần thực hiện tốt việc kết nối giữa các nhà cung cấp giải pháp ERP và người sử dụng hệ thống ERP tại doanh nghiệp; khi triển khai hệ thống ERP cần lựa chọn những nhà cung cẩp giải pháp ERP có chế độ hỗ trợ, giải đáp thắc mắc tốt cho doanh nghiệp không phải chỉ trong giai đoạn triển khai hệ thống mà cả ở giai đoạn sau triển khai, nhằm mang lại sự hài lòng cao nhất trong quá trình sử dụng hệ thống ERP của người sử dụng tại doanh nghiệp. Chỉ có như vậy mới đảm bảo doanh nghiệp thu được đầy đủ các lợi ích kế toán trong hệ thống ERP ở giai đoạn này.

\section{TÀI LIỆU THAM KHẢO}

[1] Colmenares, L., Benefits of ERP systems for accounting and financial management, Proceedings of the Acedemy of Information and Management Sciences, 13(1), 3-7, 2009.

[2] DeLone, W.H. and McLean, E.R., Information systems success measurement, Foundations and Trends® in Information Systems, 2(1), pp.1-116, 2016.

[3] Despina Galani, Efthymios Gravas, Antonios Stavropoulos, 2010. The Impact of ERP Systems on Accounting Processes. International Journal of Economics and Management Engineering, Vol 4, No 6, 774-779.

[4] Esteves, J. and Pastor, J., 1999, November. An ERP lifecycle-based research agenda. In 1st International Workshop in Enterprise Management \& Resource Planning.

[5] Edith Galy \& Mary Jane Sauceda, 2014. Post-implementation practices of ERP systems and their relationship to financial performance. Information \& Management 51,310-319.

[6] Jiwat Ram, David Corkindalea, Ming-Lu Wu, 2013. Implementation Critical Success Factors (CSFs) for ERP: Do they contribute to implementation success and post-implementation performance? International Journal of Production Economics, 144(1), 157-174.

[7] Hawking, P., Stein, A. and Foster, S., 2004, January. Revisiting ERP systems: benefit realization. In 37th Annual Hawaii International Conference on System Sciences, 2004. Proceedings of the (pp. 8-pp). IEEE.

[8] Kanellou, A., and Spathis, C., Accounting benefits and satisfaction in an ERP environment, International Journal of Accounting Information Systems, 14:209-234, 2013.

[9] Majed Al-Mashari, Abdullah Al-Mudimigh, and Mohamed Zairi, Enterprise resource planning: A taxonomy of critical factors, European Journal of Operational Research, 146 (2003) 352-364, 2003.

[10] Nguyễn Đình Thọ, Phương pháp nghiên cứu khoa học trong kinh doanh, Ân bản lần 2. Hồ Chí Minh: Nhà xuất bản tài chính, 2014.

[11] Petter, S., DeLone, W. and McLean, E., Measuring information systems success: models, dimensions, measures, and interrelationships. European journal of information systems, 17(3), pp.236-263, 2008.

[12] Pedro Ruivo, Tiago Oliveira, Miguel Neto, 2014. Examine ERP post-implementation stages of use and value: Empirical evidence from Portuguese SMEs. International Journal of Accounting Information Systems 15, 166184. 
[13] Sedera, D., \& Gable, G., A factor and structural equation analysis of the enterprise systems success measurement model, ICIS 2004 Proceedings, 36, 2004.

[14] Shih-Wei Chou and Yu-Chieh Chang, The implementation factors that influence the ERP (enterprise resource planning) benefits, Decision Support Systems, 46 (2008) 149-157, 2008.

[15] Spathis C, Enterprise systems implementation and accounting benefits, J Enterp Inf Manag, 19(1): 67-82, 2006.

[16] Spathis C, Constantinides S, Enterprise resource planning systems' impact on accounting processes, Bus Process Manag J, 10(3): 234-247, 2004.

[17] Spathis, C., and Ananiadis, J., Assessing the benefits of using an enterprise system in accounting information and management, Journal of enterprise Information Management, 18(2), 195-210, 2005.

[18] WILliam H. DeLone and EPHRAIM R. McLean, The DeLone and McLean Model of Information Systems Success: A Ten-Year Update, Journal of Management Information Systems, Vol. 19, no. 4, pp. 9-30, 2003.

[19] Young Mok Haa, Hyung Jun Ahn, 2014. Factors affecting the performance of Enterprise Resource Planning (ERP) systems in the post-implementation stage. Behaviour \& Information Technology, Vol. 33, No. 10, 10651081.

Ngày nhận bài: 23/07/2019

Ngày chấp nhận đăng: 22/11/2019 